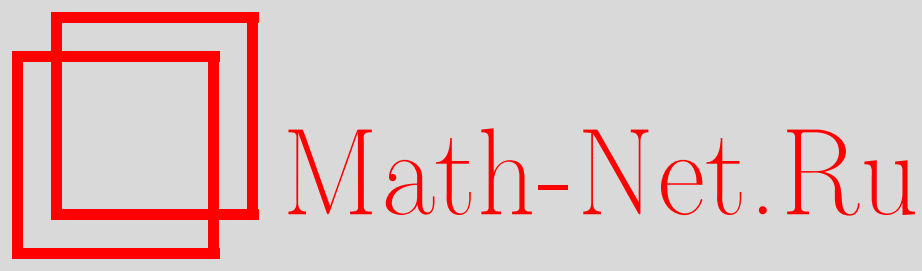

М. Ю. Малышев, Е. В. Прохватилов, Квантовая хромодинамика на световом фронте с нулевыми модами, моделирующими вакуум, ТМФ, 2011, том 169, номер 2, 272284

DOI: https://doi.org/10.4213/tmf6728

Использование Общероссийского математического портала Math-Net.Ru подразумевает, что вы прочитали и согласны с пользовательским соглашением http: //www.mathnet.ru/rus/agreement

Параметры загрузки:

IP: 3.80 .253 .173

26 апреля 2023 г., 09:41:21

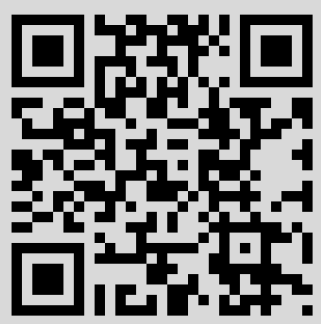




\title{
ФИЗИКА
}

Том 169, № 2

ноябрь, 2011

(C) 2011 г.

М. Ю. Малышев*, Е. В. Прохватилов*

\section{КВАНТОВАЯ ХРОМОДИНАМИКА НА СВЕТОВОМ ФРОНТЕ С НУЛЕВЫМИ МОДАМИ, МОДЕЛИРУЮЩИМИ ВАКУУМ}

\begin{abstract}
Представлена попытка построить гамильтониан квантовой хромодинамики на световом фронте, включающий полуфеноменологическое описание вакуумных эффектов. В рамках данного подхода теория на световом фронте получается путем предельного перехода от теории, сформулированной на пространственноподобных плоскостях, близких к световому фронту. Чтобы нулевые моды Фурье полей по координате вдоль светового конуса остались независимыми динамическими переменными на световом фронте, предельный переход осуществляется по-разному для нулевых и ненулевых мод. При этом нулевые моды моделируют вакуум на световом фронте и позволяют ввести полуфеноменологическое описание вакуумных эффектов. Для калибровочно-инвариантной регуляризации вводится решетка в пространстве "поперечных" координат и калибровочно-инвариантное ограничение компоненты импульса вдоль светового конуса. Дано новое описание полевых переменных на решетке: для глюонных нулевых мод используются унитарные матрицы, относящиеся к ребрам решетки, а для ненулевых мод - эрмитовы матрицы, относящиеся к соответствующим узлам решетки.
\end{abstract}

Ключевые слова: калибровочные поля, квантовая хромодинамика, световой фронт, гамильтонов подход, поперечная решетка.

\section{1. ВВЕДЕНИЕ}

Формулировка квантовой теории поля на световом фронте (СФ) была инициирована Дираком еще в 1949 г. [1]. Вместо обычных лоренцевых координат $x^{0}, x^{1}$, $x^{2}, x^{3}$ вводятся так называемые координаты СФ

$$
x^{ \pm}=\frac{x^{0} \pm x^{3}}{\sqrt{2}}, \quad x^{\perp}=\left(x^{1}, x^{2}\right)=x^{k},
$$

где $x^{+}$играет роль времени, и СФ определяется условием $x^{+}=0$. Эта формулировка ведет к возможности простого описания вакуумного состояния как состояния

${ }^{*}$ Санкт-Петербургский государственный университет, Санкт-Петербург, Россия. E-mail: mimalysh@yandex.ru, Evgeni.Prokhvat@pobox.spbu.ru 
с наименьшим собственным значением $p_{-}=0$ оператора импульса $P_{-}$(генератора сдвига по координате $\left.x^{-}\right)$:

$$
P_{-}=\frac{P_{0}-P_{3}}{\sqrt{2}}, \quad P_{-} \geqslant 0, \quad p_{0} \geqslant 0, \quad p^{2} \geqslant 0
$$

В обычном квантовании на поверхности фиксированного лоренцева времени именно сложность описания вакуумного состояния является основной трудностью при попытках непертурбативного решения уравнения Шредингера для поля, поэтому квантование на СФ позволяет развивать непертурбативный подход к расчету спектра масс связанных состояний поля, в том числе спектра адронов в квантовой хромодинамике (KХД) путем решения задачи на собственные значения гамильтониана $P_{+}=\left(P_{0}+P_{3}\right) / \sqrt{2}$ на СФ в пространстве Фока на СФ.

Каноническая формулировка теории поля на СФ сталкивается с трудностями рассмотрения нулевых мод Фурье поля по координате $x^{-}$. Например, кинетический член в лагранжиане $L(x)$ для скалярного поля $\phi(x)$ имеет в координатах СФ следующий вид: $L=\partial_{+} \phi \partial_{-} \phi+\cdots$, где выделено слагаемое, содержащее производную по времени $x^{+}$. Нулевая мода $\left(p_{-}=0\right)$, т. е. поле, не зависящее от $x^{-}$, не содержится в этом члене, поэтому канонически сопряженный к ней импульс обращается в ноль, и нулевая мода не является независимой динамической переменной, в отличие от остальных мод. Наряду с этой особенностью возникают сингулярности в выражении для гамильтониана при $p_{-}=0$.

Обычно применяют два типа регуляризации: регуляризацию $\left|p_{-}\right| \geqslant \varepsilon>0$ и так называемую DLCQ-регуляризацию (дискретизованное на световом конусе квантование) [2], [3], т. е. ограничение пространства $\left|x^{-}\right| \leqslant L$ по переменной $x^{-}$при периодических граничных условиях на (калибровочно-инвариантные) функции поля. Однако первая регуляризация не имеет лоренцевой и калибровочной симметрии и исключает нулевые по $x^{-}$моды, которые важны для правильного описания вакуумных эффектов. С другой стороны, DLCQ-регуляризация также нарушает лоренцеву симметрию, но сохраняет калибровочную инвариантность. Импульс $p_{-}$становится дискретным из-за периодических граничных условий. Четко отделенные нулевые моды могут быть выражены через другие моды путем решения канонических связей. Однако для большинства моделей эти связи очень сложны, и задача практически неразрешима [4], [5]. Нарушение лоренцевой и калибровочной симметрий в этих регуляризациях ведет к затруднениям при перенормировке теории, а также к возможной ее неэквивалентности обычной формулировке в лоренцевых координатах. В рамках теории возмущений было показано, что для восстановления эквивалентности необходимо ввести в регуляризованный гамильтониан формализма на СФ дополнительные члены типа “контрчленов", вводимых при перенормировке теории [6].

Чтобы исследовать проблему описания вакуумных эффектов на СФ, мы используем хорошо известную модель калибровочного поля в двумерном пространстве-времени, а именно $(1+1)$-мерную квантовую электродинамику $($ КЭД $(1+1))$. Эта модель может быть преобразована в модель скалярного поля с неполиномиальным взаимодействием, которая намного проще для рассмотрения на СФ, чем исходная калибровочная теория. В результате проделанного ранее исследования [7], [8] были

4 Теоретическая и математическая физика, т. 169, № 2, 2011 г. 
найдены члены, которые необходимо добавить к каноническому выражению для гамильтониана на СФ, чтобы сделать формулировку на СФ эквивалентной обычной формулировке в лоренцевых координатах. Эти члены содержат всю информацию о вакуумных конденсатах и зависят от нулевых мод.

Было также замечено, что аналогичные вакуумные эффекты для спектра масс в КЭД $(1+1)$ можно приближенно получить в рамках полуфеноменологического подхода, связанного с предельным переходом к гамильтониану на СФ от гамильтонианов на пространственноподобных плоскостях, близких к СФ. При этом вводятся координаты, характеризующие такие пространственноподобные плоскости и совпадающие в пределе с координатами СФ. Далее, по той координате, которая совпадает в пределе с координатой $x^{-}$, вводится ограничение пространства (с аналогичным параметром $L$ ) и соответствующие периодические граничные условия. Пока параметр $L$ конечен и фиксирован, предельный переход совершается так, чтобы нулевые моды оставались независимыми динамическими переменными, какими они были до перехода на СФ. В этом смысле для нулевых мод предельный переход на СФ "замораживается". В результате нулевые моды, моделирующие "инфракрасную" динамику, дают полуфеноменологический способ описания вакуумных конденсатов и правильного описания спектра масс в пределе снятия регуляризации [9].

Попытка применить аналогичный полуфеноменологический способ для приближенного описания вакуумных эффектов в КХД на СФ была изложена ранее в работе [10], однако использованная в данной работе регуляризация не имела калибровочной симметрии. В настоящей работе предлагается калибровочно-инвариантная регуляризация, удобная для рассмотрения предельного перехода на СФ. Мы используем конечную (с периодическими граничными условиями) решетку в пространстве "поперечных" координат $x^{1}, x^{2}$ и калибровочно-инвариантный аналог "ультрафиолетового" ограничения импульса $p_{-}$. В предлагаемой регуляризации только нулевые моды глюонного поля отнесены к ребрам решетки и описываются унитарными матрицами, остальные поля отнесены к узлам решетки.

Пока регуляризация не снята, лоренцева симметрия нарушена этой регуляризацией. Вакуумное состояние, заданное как собственное состояние оператора $P_{-}$с минимальным собственным значением $p_{-}=0$, определено неоднозначно из-за нулевых мод. Минимум оператора $P_{-}$может не соответствовать минимуму гамильтониана $P_{+}$. Мы принимаем приближение, в котором вакуум определяется как состояние, минимизирующее проекцию (т. е. редукцию) гамильтониана $P_{+}$на собственное подпространство с нулевым значением импульса $P_{-}$. Такая проекция зависит только от нулевых мод (которые остаются независимыми динамическими переменными на СФ после вышеупомянутого предельного перехода при конечном $L$ ) и выглядит подобно гамильтониану калибровочной теории на пространственной решетке в $(2+1)$-измерениях (где координата $x^{+}$может быть формально отождествлена с лоренцевым временем). Чтобы найти массы адронов, необходимо строить состояния с конечным значением импульса $P_{-}$, используя над данным вакуумом базис пространства Фока на СФ, связанный с ненулевыми модами.

Мы надеемся, что лоренцева инвариантность может быть восстановлена в пределе снятия регуляризации, и поэтому вакуумное состояние, определенное путем минимизации редукции гамильтониана $P_{+}$на собственное подпространство оператоpa $P_{-}$с собственным значением $p_{-}=0$, совпадет в пределе снятия регуляризации с 
собственным состоянием оператора $P_{+}$, отвечающим его минимальному собственному значению. Окончательный ответ может быть получен в результате дальнейших численных расчетов.

\section{2. ГАМИЛЬТОНИАН КХД В КООРДИНАТАХ, БЛИЗКИХ К КООРДИНАТАМ СФ}

Начнем с лагранжевой формулировки в координатах $y^{\mu}=\left(y^{0}, y^{1}, y^{2}, y^{3}\right)$, аппроксимирующих координаты СФ:

$$
y^{0}=x^{+}+\frac{\eta^{2}}{2} x^{-}, \quad y^{3}=x^{-}, \quad y^{\perp}=x^{\perp} .
$$

Предельный переход к теории на СФ соответствует пределу $\eta \rightarrow 0$. В этих координатах мы имеем следующую плотность лагранжиана КХД (с калибровочной группой $S U(N))[10]:$

$$
\begin{aligned}
L(y)=\operatorname{Tr} & \left\{F_{03}^{2}(y)+2 F_{0 k}(y) F_{3 k}(y)+\eta^{2} F_{0 k}^{2}(y)-F_{12}^{2}(y)\right\}+ \\
& +i \sqrt{2} \psi_{+}^{+}(y) D_{0} \psi_{+}(y)+\frac{i \eta^{2}}{\sqrt{2}} \psi_{-}^{+}(y) D_{0} \psi_{-}(y)+i \sqrt{2} \psi_{-}^{+}(y) D_{3} \psi_{-}(y)+ \\
& +i \psi_{-}^{+}(y)\left(D_{\perp}-m\right) \psi_{+}(y)+i \psi_{+}^{+}(y)\left(D_{\perp}+m\right) \psi_{-}(y),
\end{aligned}
$$

где $F_{\mu \nu}(y)=\partial_{\mu} A_{\nu}(y)-\partial_{\nu} A_{\mu}(y)-i g\left[A_{\mu}(y), A_{\nu}(y)\right] ; A_{\mu}(y)$ - векторные потенциалы глюонного поля, отнесенные к координатам $y^{\mu} ; D_{\mu}=\partial_{\mu}-i g A_{\mu}(y)$,

$$
D_{\perp}=\sum_{k=1,2} \sigma_{k} D_{k}
$$

$\sigma_{k}$ - матрицы Паули, $g$ - константа связи, $m$ - масса поля фермионов (кварков), описываемого биспинором $\psi=\left(\begin{array}{c}\psi_{+} \\ \psi_{-}\end{array}\right)$, который имеет компоненты $\psi_{ \pm, r}^{j}$ со спиновыми индексами $r= \pm 1 / 2$ и $S U(N)$-индексами $j=1,2, \ldots, N$. Эти спиноры отнесены к системе координат $x^{\mu}$ и соответствуют выбору матриц Дирака $\gamma^{\mu}$ в координатах $x^{\mu}$ в следующем виде:

$$
\gamma^{0}=i\left(\begin{array}{cc}
0 & -I \\
I & 0
\end{array}\right), \quad \gamma^{3}=i\left(\begin{array}{cc}
0 & I \\
I & 0
\end{array}\right), \quad \gamma^{k}=i\left(\begin{array}{cc}
\sigma_{k} & 0 \\
0 & \sigma_{k}
\end{array}\right) .
$$

Введем решетку по координатам $y^{1}=x^{1}, y^{2}=x^{2}$ с параметром $a$ (расстоянием между соседними узлами решетки), а также решетку по координате $y^{0}$ с параметром $a_{0}$ (в дальнейшем мы будем устремлять его к нулю в соответствующей "трансфер-матрице" по $\left.y^{0}\right)$. Координату $y^{3}$ ограничим условием $\left|y^{3}\right| \leqslant L$ и при этом поля подчиним периодическим граничным условиям по $y^{3}$.

Компоненту глюонного поля $A_{3}(y)$ и кварковые поля мы оставляем без изменения и относим к узлам решетки. Для поперечных компонент глюонного поля вводим новые специальные переменные в виде комплексных $(N \times N)$-матриц следующего вида (для модели с калибровочной группой $S U(N)$ ):

$$
M_{\alpha}(y)=\left(I+i g a_{\alpha} \tilde{A}_{\alpha}(y)\right) U_{\alpha}(y), \quad \alpha=0,1,2, \quad a_{1}=a_{2}=a,
$$


где $U_{\alpha}(y)$ - унитарные $(N \times N)$-матрицы, отнесенные к ребрам решетки $\left(y-a_{\alpha} e_{\alpha}, y\right)$, векторы $e_{\alpha}$ - единичные векторы вдоль осей $y^{\alpha}$, а $\tilde{A}_{\alpha}(y)-$ эрмитовы $(N \times N)$-матрицы, отнесенные к соответствующим узлам решетки. Для этих переменных мы определяем закон калибровочных преобразований следующим образом:

$$
\tilde{A}_{\alpha}(y) \rightarrow \Omega(y) \tilde{A}_{\alpha}(y) \Omega^{+}(y), \quad U_{\alpha}(y) \rightarrow \Omega(y) U_{\alpha}(y) \Omega^{+}\left(y-a_{\alpha} e_{\alpha}\right),
$$

где $\Omega(y)$ - матрица калибровочного преобразования. Для матриц $M_{\alpha}(y)$ преобразование имеет следующий вид:

$$
M_{\alpha}(y) \rightarrow \Omega(y) M_{\alpha}(y) \Omega^{+}\left(y-a_{\alpha} e_{\alpha}\right) .
$$

При этом для переменных $A_{3}$ и $\psi$ мы сохраняем обычный закон преобразования

$$
A_{3}(y) \rightarrow \Omega(y) A_{3}(y) \Omega^{+}(y)+\frac{i}{g} \Omega(y) \partial_{3} \Omega^{+}(y), \quad \psi(y) \rightarrow \Omega(y) \psi(y) .
$$

Определим оператор $D_{3}$ следующими равенствами:

$$
\begin{aligned}
D_{3} \tilde{A}_{\alpha}(y) & =\partial_{3} \tilde{A}_{\alpha}(y)-i g\left[A_{3}(y), \tilde{A}_{\alpha}(y)\right] \\
D_{3} U_{\alpha}(y) & =\partial_{3} U_{\alpha}(y)-i g A_{3}(y) U_{\alpha}(y)+i g U_{\alpha}(y) A_{3}\left(y-a_{\alpha} e_{\alpha}\right), \\
D_{3} M_{\alpha}(y) & =\partial_{3} M_{\alpha}(y)-i g A_{3}(y) M_{\alpha}(y)+i g M_{\alpha}(y) A_{3}\left(y-a_{\alpha} e_{\alpha}\right), \\
D_{3} \psi(y) & =\left(\partial_{3}-i g A_{3}(y)\right) \psi(y) .
\end{aligned}
$$

Эти равенства обеспечивают перестановочность оператора $D_{3}$ с калибровочными преобразованиями. Доопределим матрицы $U_{\alpha}(y)$ следующим калибровочно-инвариантным условием: $D_{3} U_{\alpha}(y)=0$, а для матриц $\tilde{A}_{\alpha}(y)$ потребуем отсутствия в них части, удовлетворяющей условию $D_{3} \tilde{A}_{\alpha}(y)=0$. В калибровке $A_{3}=0$ эти условия просто означают разделение нулевых и ненулевых мод Фурье по $y^{3}$ матриц $M_{\alpha}(y)$, где $U_{\alpha}(y)$ и $i g a_{\alpha} \tilde{A}_{\alpha}(y) U_{\alpha}(y)$ описывают нулевые и ненулевые моды соответственно.

В пределе непрерывного пространства $a, a_{0} \rightarrow 0$ потребуем, чтобы в калибровке $A_{3}=0$ для $U_{\alpha}(y)$ выполнялось следующее условие:

$$
U_{\alpha}(y) \rightarrow e^{i g a_{\alpha} A_{\alpha 0}(y)} \rightarrow I+i g a_{\alpha} A_{\alpha 0}(y),
$$

где $A_{\alpha 0}(y)$ - нулевая мода поля $A_{\alpha}(y)$ в непрерывном пространстве. Для поля $\tilde{A}_{\alpha}(y)$ потребуем, чтобы оно стремилось к части поля $A_{\alpha}(y)$, отвечающей ненулевым модам. Тогда при всех $A_{3}$ для матриц $M_{\alpha}(y)$ получаем следующее соотношение:

$$
M_{\alpha}(y) \rightarrow I+i g a_{\alpha} A_{\alpha}(y)+O\left(\left(a_{\alpha} g\right)^{2}\right) .
$$

Это позволяет определить решеточный аналог полей $F_{\mu \nu}(y)$ теории в непрерывном пространстве следующим образом:

$$
\begin{aligned}
& G_{12}(y)=-\frac{1}{g a^{2}}\left(M_{1}(y) M_{2}\left(y-a e_{1}\right)-M_{2}(y) M_{1}\left(y-a e_{2}\right)\right), \\
& G_{0 k}(y)=\frac{1}{g a a_{0}}\left(M_{k}(y) M_{0}\left(y-a e_{k}\right)-M_{0}(y) M_{k}\left(y-a_{0} e_{0}\right)\right), \quad k=1,2, \\
& G_{3 \alpha}(y)=\frac{1}{g a_{\alpha}} D_{3} M_{\alpha}(y) .
\end{aligned}
$$

При $a, a_{0} \rightarrow 0$ получаем $G_{\mu \nu}(y) \rightarrow i F_{\mu \nu}(y)$. 
При калибровочных преобразованиях имеем

$$
\begin{aligned}
& G_{3 \alpha}(y) \rightarrow \Omega(y) G_{3 \alpha}(y) \Omega^{+}\left(y-a_{\alpha} e_{\alpha}\right), \\
& G_{\alpha \beta}(y) \rightarrow \Omega(y) G_{\alpha \beta}(y) \Omega^{+}\left(y-a_{\alpha} e_{\alpha}-a_{\beta} e_{\beta}\right) .
\end{aligned}
$$

Далее, мы можем ввести калибровочно-инвариантный аналог ультрафиолетового обрезания по $p_{3}$, используя обрезание $\left|q_{3}\right| \leqslant \Lambda$ по собственным значениям $q_{3}$ оператора $D_{3}$. Это условие позволяет придать регуляризации теории полностью калибровочно-инвариантный вид.

Выпишем действие регуляризованной теории в следующем виде:

$$
\begin{aligned}
S(\eta)= & \sum_{y^{\perp}, y^{0}} \int_{-L}^{L} d y^{3} a^{2} a_{0}\left\{\operatorname { T r } \left(G_{03}^{+}(y) G_{03}(y)+\eta^{2} G_{0 k}^{+}(y) G_{0 k}(y)+G_{0 k}^{+}(y) G_{3 k}(y)+\right.\right. \\
& \left.+G_{3 k}^{+}(y) G_{0 k}(y)-G_{12}^{+}(y) G_{12}(y)\right)-\frac{i}{a_{0} \sqrt{2}}\left(\psi_{+}^{+}(y) M_{0}(y) \psi_{+}\left(y-a_{0} e_{0}\right)-\text { э. c. }\right)- \\
& -\frac{i \eta^{2}}{2 \sqrt{2} a_{0}}\left(\psi_{-}^{+}(y) M_{0}(y) \psi_{-}\left(y-a_{0} e_{0}\right)-\text { э. c. }\right)+i \sqrt{2} \psi_{-}^{+}(y) D_{3} \psi_{-}(y)- \\
& -\frac{i}{2 a}\left(\psi_{-}^{+}(y) M_{k}(y) \sigma_{k} \psi_{+}\left(y-a e_{k}\right)+\psi_{+}^{+}(y) M_{k}(y) \sigma_{k} \psi_{-}\left(y-a e_{k}\right)-\text { э. c. }\right)- \\
& \left.-i m\left(\psi_{-}^{+}(y) \psi_{+}(y)-\psi_{+}^{+}(y) \psi_{-}(y)\right)\right\} .
\end{aligned}
$$

Далее для краткости будет принята следующая сокращенная запись аргументов у функций поля: поле в точке $y$ пишется без указания на аргумент, а поле в смещенной точке $y \pm a_{\alpha} e_{\alpha}$ пишется с указанием только на величину и направление сдвига, например $f(y)=f, f\left(y \pm a_{\alpha} e_{\alpha}\right)=f\left( \pm a_{\alpha}\right)$.

При конечном значении $\eta \neq 0$ нулевые моды - независимые динамические переменные. Чтобы сохранить это свойство в пределе $\eta \rightarrow 0$, модифицируем действие, фиксируя параметр $\eta$ равным $\eta_{0} \neq 0$ в соответствующих членах действия:

$$
\begin{aligned}
& S\left(\eta, \eta_{0}\right)=S(\eta)+2 L\left(\eta_{0}^{2}-\eta^{2}\right) \sum_{y^{\perp}, y^{0}}\left(\frac { 1 } { g ^ { 2 } a _ { 0 } } \operatorname { T r } \left\{\left(U_{k} U_{0}\left(-a_{k}\right)-U_{0} U_{k}\left(-a_{0}\right)\right) \times\right.\right. \\
& \left.\left.\quad \times\left(U_{0}^{+}\left(-a_{k}\right) U_{k}^{+}-U_{k}^{+}\left(-a_{0}\right) U_{0}^{+}\right)\right\}-\frac{i}{2 \sqrt{2} a_{0}}\left(\psi_{-, 0}^{+} U_{0} \psi_{-, 0}\left(-a_{0}\right)-\text { э. с. }\right)\right) .
\end{aligned}
$$

Здесь $\psi_{-, 0}$ обозначает моду фермионного поля, которая определяется равенством $D_{3} \psi_{-, 0}=\left(\partial_{3}-i g A_{3}\right) \psi_{-, 0}=0$.

Для построения гамильтониана применим изложенный в работе [11] метод получения гамильтониана из соответствующей "трансфер-матрицы” на решетке по времени $y^{0}$. С этой целью выберем $A_{3}=0, U_{0}=I$ как калибровочные условия. Заметим, что, выбирая для полей периодические граничные условия по $y^{3}$, мы ограничиваем такими условиями класс калибровочных преобразований. При этом условие $A_{3}=0$ не только ограничивает калибровочную свободу, но и сужает общий класс физически допустимых полей [4]. Однако в случае КЭД $(1+1)$ было замечено [9], что можно получить хорошее полуфеноменологическое описание спектра масс на СФ даже с так ограниченным классом полей. Поэтому здесь мы рассматриваем этот более простой вариант. 
Полагая $A_{3}=0, U_{0}=I$, “глюонную" часть действия можно переписать следующим образом:

$$
\begin{aligned}
& S_{\mathrm{G}}\left(\eta, \eta_{0}\right)=\sum_{y^{\perp}, y^{0}} \int_{-L}^{L} d y^{3} a^{2} a_{0} \operatorname{Tr}\left\{\left(\partial_{3} \tilde{A}_{0}\right)^{2}-\frac{i}{g a a_{0}} \partial_{3} \tilde{A}_{k}\left[\left(I+i g a \tilde{A}_{k}\right) \times\right.\right. \\
& \left.\quad \times\left(I+i g a_{0} U_{k} \tilde{A}_{0}\left(-a_{k}\right) U_{k}^{+}\right)-\left(I+i g a_{0} \tilde{A}_{0}\right)\left(I+i g a \tilde{A}_{k}\left(-a_{0}\right)\right) U_{k}\left(-a_{0}\right) U_{k}^{+}-\text {э. c. }\right]+ \\
& \quad+\frac{\eta^{2}}{g^{2} a^{2} a_{0}^{2}}\left[\left(I+g^{2} a_{0}^{2} U_{k} \tilde{A}_{0}^{2}\left(-a_{k}\right) U_{k}^{-1}\right)\left(I+g^{2} a^{2} \tilde{A}_{k}^{2}\right)-\left[U_{k} U_{k}^{+}\left(-a_{0}\right) \times\right.\right. \\
& \left.\quad \times\left(I-i g a \tilde{A}_{k}\left(-a_{0}\right)\right)\left(I-i g a_{0} \tilde{A}_{0}\right)\left(I+i g a \tilde{A}_{k}\right)\left(I+i g a_{0} U_{k} \tilde{A}_{0}\left(-a_{k}\right) U_{k}^{+}\right)+\text {э. c. }\right]+ \\
& \left.\left.\quad+\left(I+g^{2} a^{2} \tilde{A}_{k}^{2}\left(-a_{0}\right)\right)\left(I+g^{2} a_{0}^{2} \tilde{A}_{0}^{2}\right)\right]-G_{12}^{+} G_{12}\right\}+ \\
& \quad+2 L\left(\eta_{0}^{2}-\eta^{2}\right) \sum_{y^{\perp}, y^{0}}\left(\frac{1}{g^{2} a_{0}} \operatorname{Tr}\left\{2-\left(U_{k} U_{k}^{+}\left(-a_{0}\right)+\text { э. с. }\right)\right\}\right) .
\end{aligned}
$$

Произведение $U_{k} U_{k}^{+}\left(-a_{0}\right)$, входящее в это выражение, отвечает сдвигу вдоль оси времени на шаг решетки, что используется при построении соответствующей трансфер-матрицы и гамильтониана в пределе $a_{0} \rightarrow 0$ по методу работы [11]. Применяя этот метод для глюонной части действия [12], получаем следующее выражение для глюонной части гамильтониана в пределе $a_{0} \rightarrow 0$ (с точностью до членов порядка $\left.\eta^{2}\right)$ :

$$
\begin{aligned}
H_{\mathrm{G}}= & \sum_{y^{\perp}, k}\left[\frac{g^{2}}{4 L \eta_{0}^{2}}\left(\pi_{k}^{a}-\frac{1}{2} \int_{-L}^{L} d y^{3} f^{a b c} \widetilde{\Pi}_{k}^{b} \tilde{A}_{k}^{c}\right)^{2}+\frac{1}{2 \eta^{2} a^{2}} \int_{-L}^{L} d y^{3}\left(\widetilde{\Pi}_{k}^{a}-a^{2} \partial_{3} \tilde{A}_{k}^{a}\right)^{2}+\right. \\
& \left.+\int_{-L}^{L} d y^{3} \widetilde{\Pi}_{k}^{a} \operatorname{Tr}\left\{\lambda^{a}\left(\frac{\tilde{A}_{0}-U_{k} \tilde{A}_{0}\left(-a_{k}\right) U_{k}^{+}}{a}-i g\left[\tilde{A}_{k},\left(\tilde{A}_{0}+U_{k} \tilde{A}_{0}\left(-a_{k}\right) U_{k}^{+}\right)\right]\right)\right\}\right]- \\
& -a^{2} \sum_{y^{\perp}} \int_{-L}^{L} d y^{3} \operatorname{Tr}\left\{\left(\partial_{3} \tilde{A}_{0}\right)^{2}-G_{12}^{+} G_{12}\right\}+O\left(\eta^{2}\right) .
\end{aligned}
$$

Здесь $\widetilde{\Pi}_{k}^{a}$ и $\tilde{A}_{k}^{a}=\operatorname{Tr}\left(\lambda^{a} \tilde{A}_{k}\right)-$ канонически сопряженные пары переменных, $\lambda^{a}-$ аналоги матриц Гелл-Манна для группы $S U(N)$,

$$
\left[\frac{\lambda^{a}}{2}, \frac{\lambda^{b}}{2}\right]=i f a b c \frac{\lambda^{c}}{2},
$$

$f^{a b c}$ - структурные константы, $a, b, c=1, \ldots, N^{2}-1$. Для нулевых мод роль канонических переменных играют переменные $U_{k}, \pi_{k}^{a}$, которые удовлетворяют следующим перестановочным соотношениям (при $\left.y^{0}=y^{0^{\prime}}\right)$ :

$$
\begin{aligned}
& {\left[\pi_{k}^{a}(y), U_{k^{\prime}}\left(y^{\prime}\right)\right]=-\delta_{k k^{\prime}} \delta_{y^{\perp} y^{\perp^{\prime}}} \frac{\lambda^{a}}{2} U_{k}(y),} \\
& {\left[\pi_{k}^{a}(y), \pi_{k^{\prime}}^{b}\left(y^{\prime}\right)\right]=i \delta_{k k^{\prime}} \delta_{y^{\perp} y^{\perp^{\prime}}} f^{a b c} \pi_{k}^{c}(y) .}
\end{aligned}
$$

Рассмотрим зависящую от фермионных переменных часть действия. Нулевые моды глюонных полей, описываемые унитарными матрицами, входят в эту часть 
без производных по времени, поэтому можно не вводить дополнительной решетки по времени и переходить к рассматриваемой части гамильтониана, применяя обычный канонический формализм.

Введем нормированные фермионные переменные

$$
\chi=2^{1 / 4} \psi_{+}, \quad \xi=2^{-1 / 4} \eta\left(\psi_{-}-\frac{1}{2 L} \int_{-L}^{L} d y^{3} \psi_{-}\right), \quad \xi_{0}=2^{-1 / 4} \frac{a \eta_{0}}{\sqrt{2 L}} \int_{-L}^{L} d y^{3} \psi_{-} .
$$

Тогда часть действия и гамильтониан, зависящие от фермионных переменных, записываются в следующем виде (при $\left.A_{3}=0\right)$ :

$$
\begin{aligned}
S_{\psi}= & a^{2} \sum_{y^{\perp}} \int d y^{0} \int_{-L}^{L} d y^{3}\left\{i \chi^{+} D_{0} \chi+i \xi^{+} D_{0} \xi+\left(\frac{i \eta}{a \sqrt{2 L} \eta_{0}} \xi_{0}^{+} D_{0} \xi+\text { э. с. }\right)+\right. \\
& +\frac{i}{2 L a^{2}} \xi_{0}^{+} \partial_{0} \xi_{0}+\frac{2 i}{\eta^{2}} \xi^{+} \partial_{3} \xi-\frac{i}{2 a}\left(\left(\frac{\xi^{+}}{\eta}+\frac{\xi_{0}^{+}}{\sqrt{2 L} \eta_{0} a}\right) M_{k} \sigma_{k} \chi\left(-a_{k}\right)-\text { э. с. }\right)- \\
& -\frac{i}{2 a}\left(\chi^{+} M_{k} \sigma_{k}\left(\frac{\xi\left(-a_{k}\right)}{\eta}+\frac{\xi_{0}\left(-a_{k}\right)}{\sqrt{2 L} \eta_{0} a}\right)-\text { э. c. }\right)- \\
& \left.-i m\left(\left(\frac{\xi^{+}}{\eta}+\frac{\xi_{0}^{+}}{\sqrt{2 L} \eta_{0} a}\right) \chi-\text { э.c. }\right)\right\}, \\
H_{\psi}= & a^{2} \sum_{y^{\perp}} \int_{-L}^{L} d y^{3}\left\{-g\left(\chi^{+} A_{0} \chi+\xi^{+} A_{0} \xi+\left(\frac{\eta}{a \sqrt{2 L} \eta_{0}} \xi_{0}^{+} A_{0} \xi+\text { э. c. }\right)+\right.\right. \\
& \left.+\frac{1}{2 L a^{2}} \xi_{0}^{+} A_{0} \xi_{0}\right)-\frac{2 i}{\eta^{2}} \xi^{+} \partial_{3} \xi+\frac{i}{2 a}\left(\left(\frac{\xi^{+}}{\eta}+\frac{\xi_{0}^{+}}{\sqrt{2 L} \eta_{0} a}\right) M_{k} \sigma_{k} \chi\left(-a_{k}\right)-\text { э. с. }\right)+ \\
& +\frac{i}{2 a}\left(\chi^{+} M_{k} \sigma_{k}\left(\frac{\xi\left(-a_{k}\right)}{\eta}+\frac{\xi_{0}\left(-a_{k}\right)}{\sqrt{2 L} \eta_{0} a}\right)-\text { э. c. }\right)+ \\
& \left.+i m\left(\left(\frac{\xi^{+}}{\eta}+\frac{\xi_{0}^{+}}{\sqrt{2 L} \eta_{0} a}\right) \chi-\text { э. c. }\right)\right\} .
\end{aligned}
$$

Приравнивая нулю вариацию полного гамильтониана по переменной $\tilde{A}_{0}$, получаем следующее соотношение (с точностью до членов порядка $O\left(\eta^{2}\right)$ ):

$$
\begin{aligned}
a^{2} \partial_{3}^{2} \tilde{A}_{0}^{a}=\operatorname{Tr} & \left\{\lambda ^ { a } \left(\frac{U_{k}^{-1}\left(a_{k}\right) \widetilde{\Pi}_{k}\left(a_{k}\right) U_{k}\left(a_{k}\right)-\widetilde{\Pi}_{k}}{a}-i \frac{g}{2}\left[\tilde{A}_{k}, \widetilde{\Pi}_{k}\right]-\right.\right. \\
& \left.\left.-i \frac{g}{2} U_{k}^{-1}\left(a_{k}\right)\left[A_{k}\left(a_{k}\right), \Pi_{k}\left(a_{k}\right)\right] U_{k}\left(a_{k}\right)\right)\right\}+ \\
& +\frac{g a^{2}}{2}\left(\left(\chi^{+} \lambda^{a} \chi+\xi^{+} \lambda^{a} \xi\right)+\frac{\eta}{\sqrt{2 L} a \eta_{0}}\left(\xi_{0}^{+} \lambda^{a} \xi+\text { э. с. }\right)\right) .
\end{aligned}
$$

Отсюда можно найти выражение для $\tilde{A}_{0}$ через другие переменные. 
Теперь можно написать для полного гамильтониана следующее выражение:

$$
\begin{aligned}
H= & \sum_{y^{\perp}, k}\left\{\frac{g^{2}}{4 L \eta_{0}^{2}}\left(\pi_{k}^{a}-\frac{1}{2} \int_{-L}^{L} d y^{3} f^{a b c} \widetilde{\Pi}_{k}^{b} \tilde{A}_{k}^{c}\right)^{2}+\right. \\
& +a^{2} \int_{-L}^{L} d y^{3}\left[\frac{1}{2 \eta^{2} a^{4}}\left(\widetilde{\Pi}_{k}^{a}-a^{2} \partial_{3} \tilde{A}_{k}^{a}\right)^{2}+\frac{1}{2}\left(\partial_{3} \tilde{A}_{0}^{a}\right)^{2}+\operatorname{Tr}\left(G_{12}^{+} G_{12}\right)-\frac{2 i}{\eta^{2}} \xi^{+} \partial_{3} \xi+\right. \\
& +\frac{i}{2 a}\left(\left(\frac{\xi^{+}}{\eta}+\frac{\xi_{0}^{+}}{\sqrt{2 L} \eta_{0} a}\right) M_{k} \sigma_{k} \chi\left(-a_{k}\right)-\text { э. c. }\right)+ \\
& +\frac{i}{2 a}\left(\chi^{+} M_{k} \sigma_{k}\left(\frac{\xi\left(-a_{k}\right)}{\eta}+\frac{\xi_{0}\left(-a_{k}\right)}{\sqrt{2 L} \eta_{0} a}\right)-\text { э.с. }\right)+ \\
& \left.\left.+i m\left(\left(\frac{\xi^{+}}{\eta}+\frac{\xi_{0}^{+}}{\sqrt{2 L} \eta_{0} a}\right) \chi-\text { э. с. }\right)\right]\right\}+O(\eta)
\end{aligned}
$$

где величина $\partial_{3} \tilde{A}_{0}^{a}$ должна быть выражена через другие переменные согласно формуле (4). Кроме этого, нужно выразить все поля через их моды Фурье по $y^{3}$ (которые являются независимыми переменными):

$$
\begin{gathered}
\tilde{A}_{k}^{a}=\frac{1}{a \sqrt{2 L}} \sum_{n \neq 0} \frac{a_{n, k}^{a}+a_{-n, k}^{a+}}{\sqrt{2\left|p_{n}\right|}} e^{-i p_{n} y^{3}}, \quad \widetilde{\Pi}_{k}^{a}=\frac{-i a}{\sqrt{2 L}} \sum_{n \neq 0} \frac{a_{n, k}^{a}-a_{-n, k}^{a+}}{\sqrt{2}} \sqrt{\left|p_{n}\right|} e^{-i p_{n} y^{3}}, \\
\chi_{r}^{i}=\frac{1}{a \sqrt{2 L}} \sum_{n} \chi_{n r}^{i} e^{-i p_{n} y^{3}}, \quad \xi_{r}^{i}=\frac{1}{a \sqrt{2 L}} \sum_{n \neq 0} \xi_{n r}^{i} e^{-i p_{n} y^{3}}
\end{gathered}
$$

где $p_{n}=\pi n / L, n=0, \pm 1, \pm 2, \ldots$. Так определенные независимые переменные удовлетворяют следующим (ненулевым) каноническим перестановочным соотношениям (при $\left.y^{0}=y^{0^{\prime}}\right)$ :

$$
\begin{aligned}
& {\left[a_{n, k}^{a}(y), a_{n^{\prime}, k^{\prime}}^{b+}\left(y^{\prime}\right)\right]=\delta_{k k^{\prime}} \delta_{a b} \delta_{n n^{\prime}} \delta_{y^{\perp} y^{\perp^{\prime}}},} \\
& \left\{\chi_{n r}^{i}(y), \chi_{n^{\prime} r^{\prime}}^{j+}\left(y^{\prime}\right)\right\}=\left\{\xi_{n r}^{i}(y), \xi_{n^{\prime} r^{\prime}}^{j+}\left(y^{\prime}\right)\right\}=\delta_{n n^{\prime}} \delta_{i j} \delta_{r r^{\prime}} \delta_{y^{\perp} y^{\perp^{\prime}}} .
\end{aligned}
$$

К этим соотношениям следует добавить соотношения (3) для нулевых мод глюонного поля.

\section{3. ПРЕДЕЛЬНЫЙ ПЕРЕХОД К ГАМИЛЬТОНИАНУ КХД НА СВЕТОВОМ ФРОНТЕ}

Чтобы рассмотреть предельный переход на СФ $(\eta \rightarrow 0)$ для полученного гамильтониана, заметим, что при фиксированных значениях параметров $L$ и а этот гамильтониан можно разложить по степеням параметра $\eta$ следующим образом:

$$
H=\frac{1}{\eta^{2}} H_{0}+\frac{1}{\eta} H_{1}+H_{2}+O\left(\eta^{2}\right) .
$$

Имея это разложение, можно построить аналог стационарной теории возмущений по малому параметру $\eta$ :

$$
(H-E) f=0, \quad f=f_{0}+\eta f_{1}+\cdots, \quad E=\frac{1}{\eta^{2}} E_{0}+\frac{1}{\eta} E_{1}+E_{2}+O(\eta) .
$$


Заметим, что состояния $f_{0}$ соответствуют собственным состояниям гамильтониана в пределе $\eta \rightarrow 0$, т. е. на СФ. Если мы требуем ограниченности энергии $E$ в пределе $\eta \rightarrow 0$, мы должны положить $E_{0}=E_{1}=0$.

В низшем и в двух следующих порядках по $\eta$ имеем уравнения

$$
H_{0} f_{0}=0, \quad H_{0} f_{1}+H_{1} f_{0}=0, \quad H_{0} f_{2}+H_{1} f_{1}+\left(H_{2}-E_{2}\right) f_{0}=0 .
$$

Подставим в эти уравнения точные выражения для соответствующих частей гамильтониана. Выражение для $H_{0}$ в терминах мод Фурье полей имеет вид

$$
\begin{aligned}
H_{0} & =\sum_{y^{\perp}} \int_{-L}^{L} d y^{3}\left\{\frac{1}{2 a^{2}}\left(\widetilde{\Pi}_{k}^{a}-a^{2} \partial_{3} \tilde{A}_{k}^{a}\right)^{2}-2 i a^{2} \xi^{+} \partial_{3} \xi\right\}= \\
& =2 \sum_{y^{\perp}} \sum_{n>0}\left\{\left|p_{n}\right|\left(\sum_{a} a_{-n, k}^{a+} a_{-n, k}^{a}+\sum_{i}\left(\xi_{-n}^{i+} \xi_{-n}^{i}+\xi_{n}^{i} \xi_{n}^{i+}\right)\right)\right\} .
\end{aligned}
$$

Здесь отброшена постоянная, отвечающая минимальному собственному значению $H_{0}$. Следовательно, подпространство состояний $\left\{f_{0}\right\}$ может быть определено уравнениями

$$
a_{-n, k}^{a} f_{0}=\xi_{-n}^{i} f_{0}=\xi_{n}^{i+} f_{0}=0, \quad n>0,
$$

поэтому состояния $\left\{f_{0}\right\}$ играют роль вакуума для этих мод Фурье полей.

Обозначим проектор на подпространство состояний $\left\{f_{0}\right\}$ через $\mathcal{P}_{0}$. В следующем порядке по $\eta$ получаем $f_{1}=-\left(1-\mathcal{P}_{0}\right) H_{0}^{-1}\left(1-\mathcal{P}_{0}\right) H_{1} f_{0}$. Для оставшегося уравнения достаточно рассмотреть проекцию на подпространство состояний $\left\{f_{0}\right\}$. Получаем равенство

$$
\mathcal{P}_{0}\left(H_{2}-H_{1}\left(1-\mathcal{P}_{0}\right) H_{0}^{-1}\left(1-\mathcal{P}_{0}\right) H_{1}\right) f_{0}=E_{2} f_{0} .
$$

Уравнение в такой форме можно рассматривать как задачу на собственные значения для гамильтониана на СФ, поскольку величины $E_{2}$ определяют собственные значения гамильтониана $H$ в пределе $\eta \rightarrow 0$. Таким образом, для гамильтониана на СФ можно принять следующее выражение:

$$
P_{+}=\mathcal{P}_{0}\left(H_{2}-H_{1}\left(1-\mathcal{P}_{0}\right) H_{0}^{-1}\left(1-\mathcal{P}_{0}\right) H_{1}\right) \mathcal{P}_{0}
$$

Используя соотношения (6), можно устранить в этом выражении зависимость от операторов $a_{-n, k}^{a}, \xi_{-n}^{i}, \xi_{n}^{i+}(n>0)$, принимая порядок этих операторов в гамильтониане в соответствии с формулой (5). В результате получается следующее выражение в терминах независимых переменных $a_{n k}^{a}, a_{n k}^{a^{+}}, b_{n r}^{i}, b_{n r}^{i^{+}}, d_{n r}^{i}, d_{n r}^{i^{+}}(n>0)$ и $U_{k}$, $\pi_{k}^{a}, \chi_{0}, \chi_{0}^{+}, \xi_{0}, \xi_{0}^{+}$:

$$
\begin{aligned}
P_{+}= & \sum_{x^{\perp}}\left\{\int _ { - L } ^ { L } d x ^ { - } \left[\frac{g^{2}}{8 L^{2} \eta_{0}^{2}}\left(\pi_{k}^{a}-\frac{i}{2} \sum_{n>0} f^{a b c} a_{n k}^{+b} a_{n k}^{c}\right)^{2}+\frac{a^{2}}{2}\left(F_{+-}^{a}\right)^{2}+\right.\right. \\
& +a^{2} \operatorname{Tr}\left(G_{12}^{\mathrm{LF}} G_{12}^{\mathrm{LF}}\right)+\frac{i}{2 \sqrt{2 L} \eta_{0}}\left(\xi_{0}^{+}\left(I+i g a A_{k}^{\mathrm{LF}}\right) U_{k} \sigma_{k} \chi\left(-a_{k}\right)+\right. \\
& \left.+\chi^{+}\left(I+i g a A_{k}^{\mathrm{LF}}\right) U_{k} \sigma_{k} \xi_{0}\left(-a_{k}\right)-\text { э. c. }\right)+\frac{i m a}{2 \sqrt{2 L} \eta_{0}}\left(\left[\xi_{0}^{+}, \chi\right]-\left[\chi^{+}, \xi_{0}\right]\right)- \\
& -\frac{i}{8}\left(\chi^{+}\left(-a_{k^{\prime}}\right) \sigma_{k^{\prime}} U_{k^{\prime}}^{-1}\left(I-i g a A_{k^{\prime}}^{\mathrm{LF}}\right)-\chi^{+}\left(a_{k^{\prime}}\right) \times\right.
\end{aligned}
$$




$$
\begin{aligned}
& \left.\times\left(I+i g a A_{k^{\prime}}^{\mathrm{LF}}\left(a_{k^{\prime}}\right)\right) U_{k^{\prime}}\left(a_{k^{\prime}}\right) \sigma_{k^{\prime}}+2 m a \chi^{+}\right) \times \\
& \times \partial_{-}^{-1}\left(\left(I+i g a A_{k}^{\mathrm{LF}}\right) U_{k} \sigma_{k} \chi\left(-a_{k}\right)-\sigma_{k} U_{k}^{-1}\left(a_{k}\right) \times\right. \\
& \left.\times\left(I-i g a A_{k}^{\mathrm{LF}}\left(a_{k}\right)\right) \chi\left(a_{k}\right)+2 m a \chi\right)+ \\
& \left.+\frac{g^{2}}{8 L}\left(5 N-2-\frac{4}{N}\right)\left(\sum_{n>0} \frac{1}{p_{n}}\right) \operatorname{Tr}\left(A_{k}^{\mathrm{LF}} A_{k}^{\mathrm{LF}}\right)\right]+\left(\frac{g^{2}(N-1 / N)}{16 L a^{2}} \sum_{m>0} \frac{1}{p_{m}}\right) \times \\
& \left.\times\left(\sum_{n \neq m} \frac{\chi_{n}^{+} \chi_{n}}{p_{m-n}}+\left(\sum_{n>m}+\sum_{n<-m}\right) \frac{\chi_{n}^{+} \chi_{n}}{p_{n}}\right)\right\}+ \text { const, }
\end{aligned}
$$

где

$$
\begin{aligned}
F_{+-}^{a}= & \operatorname{Tr}\left(\lambda^{a} F_{+-}\right), \\
F_{+-}= & \frac{1}{a}\left(A_{k}^{\mathrm{LF}}-\left(U_{k}^{-1} A_{k}^{\mathrm{LF}} U_{k}\right)\left(a_{k}\right)\right)- \\
& -\frac{i g}{2} \partial_{-}^{-1}\left(\left[\partial_{-} A_{k}^{\mathrm{LF}}, A_{k}^{\mathrm{LF}}\right]+\left[\partial_{-}\left(U_{k}^{-1} A_{k}^{\mathrm{LF}} U_{k}\right), U_{k}^{-1} A_{k}^{\mathrm{LF}} U_{k}\right]\left(a_{k}\right)-\chi^{+} \lambda^{a} \chi \frac{\lambda^{a}}{2}\right), \\
G_{12}^{\mathrm{LF}}(x)= & -\frac{1}{g a^{2}}\left(\left(I+i g a A_{1}^{\mathrm{LF}}\right) U_{1}\left(I+i g a A_{2}^{\mathrm{LF}}\left(-a_{1}\right)\right) U_{2}\left(-a_{1}\right)-(1 \longleftrightarrow 2)\right), \\
A_{k}^{\mathrm{LF}}(x)= & \frac{1}{a \sqrt{2 L}} \sum_{a, n>0}\left(\frac{a_{n k}^{a}\left(x^{\perp}\right) e^{-i p_{n} x^{-}}}{\sqrt{2 p_{n}}}+\ni . c .\right) \frac{\lambda^{a}}{2}, \\
\chi_{r}^{i}(x)= & \frac{1}{a \sqrt{2 L}} \sum_{n>0}\left(b_{n r}^{i}\left(x^{\perp}\right) e^{-i p_{n} x^{-}}+d_{n r}^{i^{+}}\left(x^{\perp}\right) e^{i p_{n} x^{-}}\right)+\frac{\chi_{0}}{a \sqrt{2 L}} .
\end{aligned}
$$

Расходящиеся суммы в этом выражении для гамильтониана должны быть ограничены условием $p_{-}=p_{n}=\pi n / L \leqslant \Lambda$.

Оператор импульса $P_{-}$в терминах переменных $a_{n k}^{a}, a_{n k}^{a^{+}}, b_{n r}^{i}, b_{n r}^{i^{+}}, d_{n r}^{i}, d_{n r}^{i^{+}}$имеет вид

$$
P_{-}=\sum_{x^{\perp}} \sum_{n>0} p_{n}\left(a_{n k}^{a^{+}} a_{n k}^{a}+b_{n r}^{i^{+}} b_{n r}^{i}+d_{n r}^{i^{+}} d_{n r}^{i}\right) \geqslant 0 .
$$

Операторы $a_{n k}^{a}, a_{n k}^{a^{+}}, b_{n r}^{i}, b_{n r}^{i^{+}}, d_{n r}^{i}, d_{n r}^{i^{+}}$являются операторами уничтожения и рождения в фоковском пространстве с "вакуумом", соответствующим условию $P_{-}=0$, поэтому легко найти проекцию $\left(P_{+}\right)_{0}$ гамильтониана $P_{+}$на подпространство с $P_{-}=0$ :

$$
\begin{aligned}
\left(P_{+}\right)_{0}= & \sum_{x^{\perp}}\left(\frac{g^{2}}{4 L \eta_{0}^{2}} \pi_{k}^{a} \pi_{k}^{a}+\frac{4 L}{g^{2} a^{2}} \operatorname{Re} \operatorname{Tr}\left(I-U_{12}\right)\right)+ \\
& +\frac{i}{2 a \eta_{0}}\left(\xi_{0}^{+} U_{k} \sigma_{k} \chi_{0}\left(-a_{k}\right)+\chi_{0}^{+} U_{k} \sigma_{k} \xi_{0}\left(-a_{k}\right)+m a\left[\xi_{0}^{+}, \chi_{0}\right]-\text { э. c. }\right)+\text { const. }
\end{aligned}
$$

Это выражение имеет форму гамильтониана $(2+1)$-мерной калибровочной теории на пространственной решетке с непрерывным временем $x^{+}$. Вакуумное состояние соответствует минимуму такого гамильтониана.

Можно определить состояния с конечным импульсом $p_{-}$, используя операторы рождения в вышеупомянутом пространстве Фока на СФ над этим вакуумом. 
Заметим также, что, имея выражение (7) для гамильтониана на СФ, можно найти форму действия, которая порождает этот гамильтониан непосредственно в координатах СФ. Легко проверить, что такое действие совпадает с выражением (2) (т.е. с модифицированным выражением (1)) при $\eta=0$ с добавкой тех членов из выражения (7), которые получены в результате процедуры “исключения" части мод полей в силу уравнений (6). Для ясности выпишем снова эти добавочные члены:

$$
\begin{aligned}
& -\sum_{x^{\perp}, x^{+}} \int_{-L}^{L} d x^{-}\left\{\frac{g^{2}}{8 L}\left(5 N-2-\frac{4}{N}\right) \sum_{n>0} \frac{1}{p_{n}} \operatorname{Tr}\left(A_{k}^{\mathrm{LF}} A_{k}^{\mathrm{LF}}\right)+\right. \\
& \left.\quad+\left(\frac{g^{2}(N-1 / N)}{16 L a^{2}} \sum_{m>0} \frac{1}{p_{m}}\right)\left(\sum_{n \neq m} \frac{\chi_{n}^{+} \chi_{n}}{p_{m-n}}+\left(\sum_{n>m}+\sum_{n<-m}\right) \frac{\chi_{n}^{+} \chi_{n}}{p_{n}}\right)\right\} .
\end{aligned}
$$

\section{4. ЗАКЛЮЧЕНИЕ}

Представлена попытка построить гамильтониан КХД на СФ, включающий описание вакуумных эффектов. Рассмотрение аналогичной задачи для КЭД $(1+1)$ (“массивной модели Швингера") показывает возможность полуфеноменологического подхода к решению этой задачи, который включает ограничение пространства $\left|x^{-}\right| \leqslant L$ по координате вдоль светового конуса и периодические граничные условия по $x^{-}$для функций поля. При этом нулевые и ненулевые моды Фурье по этой координате можно рассматривать отдельно и описывать вакуумные эффекты при помощи нулевых мод. Кроме того, в рамках данного подхода теория на СФ получается предельным переходом от теории на пространственноподобных плоскостях, близких к СФ. Для описания предельного перехода при фиксированном значении $L$ используется параметр $\eta$, характеризующий близость пространственноподобной гиперплоскости к СФ $(\eta \rightarrow 0)$. Чтобы нулевые моды остались независимыми динамическими переменными на СФ, предельный переход осуществляется по-разному для нулевых и ненулевых мод. Фактически предельный переход осуществляется только для ненулевых мод, пока параметр $L$ конечен и фиксирован. Тогда нулевые моды могут моделировать вакуум и приводить к вакуумным эффектам в пределе $L \rightarrow \infty$.

Калибровочно-инвариантная регуляризация для КХД включает введение решетки в пространстве "поперечных" координат $x^{1}, x^{2}$ и калибровочно-инвариантный способ ограничения компоненты импульса $p_{-}$. Введено новое описание полевых переменных на решетке. Так, для глюонных нулевых мод используются унитарные матрицы, относящиеся к ребрам решетки, а для ненулевых мод - эрмитовы матрицы, относящиеся к соответствующим узлам решетки.

Регуляризация и отделение нулевых мод от остальных мод нарушают лоренцеву симметрию в формулировке теории при конечных значениях параметра решетки и параметра $L$. Мы надеемся на восстановление этой симметрии в пределе снятия регуляризации, однако это требует дополнительного исследования, которое включает также вопросы перенормировки теории и сравнения с формулировкой в лоренцевых координатах.

Благодарности. Авторы благодарят организаторов III конференции "Модели квантовой теории поля", посвященной 70-летию со дня рождения Александра Николаевича Васильева. Авторы также благодарят В. А. Франке и С. А. Пастона за полезные обсуждения. 


\section{Список литературы}

[1] P. A. M. Dirac, Rev. Mod. Phys., 21:3 (1949), 392-398.

[2] А. М. Анненкова, В. А. Франке, Е. В. Прохватилов, Вестн. ЛГУ, 4 (1985), 80-82.

[3] S. J. Brodsky, H.-C. V. Pauli, S.S. Pinsky, Phys. Rep, 301:4-6 (1998), 299-486, arXiv: hep-ph/9705477.

[4] V. A. Franke, Yu. V. Novozhilov, E. V. Prokhvatilov, Lett. Math. Phys., 5:3 (1981), 239-245.

[5] V. A. Franke, Yu. V. Novozhilov, E. V. Prokhvatilov, Lett. Math. Phys., 5:5 (1981), 437-444.

[6] С. А. Пастон, В. А. Франке, ТМФ, 112:3 (1997), 399-416, arXiv: hep-th/9901110.

[7] С. А. Пастон, Е.В. Прохватилов, В.А. Франке, ТМФ, 131:1 (2002), 84-97, arXiv: hep-th/0302016.

[8] S. A. Paston, E. V. Prokhvatilov, V. A. Franke, On the construction of corrected light-front Hamiltonian for $Q E D_{2}$, arXiv: hep-th/0011224.

[9] Е. В. Прохватилов, В. А. Франке, ЯФ, 47 (1988), 882-883.

[10] Е. В. Прохватилов, В. А. Франке, ЯФ, 49 (1989), 1109-1117.

[11] M. Creutz, Phys. Rev. D, 15:4 (1977), 1128-1136.

[12] Е.-М. Ильгенфриц, С. А. Пастон, Г.-Ю. Пирнер, Е. В. Прохватилов, В. А. Франке, TMФ, 148:1 (2006), 89-101, arXiv: hep-th/0610020. 